\title{
Qualificação do acolhimento nos serviços de saúde bucal
}

\section{Qualification of care in oral health services}

\author{
Suzely Adas Saliba Moimaz ${ }^{1}$, Danielle Bordin ${ }^{1}$, Cristina Berger Fadel ${ }^{1,2}$, \\ Celso Bilynkievycz dos Santos², Cléa Adas Saliba Garbin, Nemre Adas Saliba ${ }^{1}$
}

\begin{abstract}
Resumo
Introdução: Objetivou-se analisar a qualidade do acolhimento no serviço de saúde bucal, considerando sua capacidade de resposta às necessidades do usuário. Metodologia: Fizeram parte do estudo transversal, quantitativo, usuários da rede pública odontológica no âmbito da Atenção Primária à Saúde (APS) de três municípios de diferentes estados brasileiros $(n=1.312)$. A obtenção dos dados deu-se através de entrevista no interior das unidades de saúde. O estudo contemplou 15 variáveis referentes ao processo de acolhimento, as quais foram analisadas preliminarmente com testes qui-quadrado. As que apresentaram associação com a variável desfecho "capacidade de resposta às necessidades do usuário" foram analisadas, posteriormente, por meio da regressão logística e teste de seleção de atributos. Resultados: Verificou-se que avaliação negativa do cumprimento das necessidades esteve estatisticamente associada ao atendimento do cirurgião-dentista e equipe; disponibilidade para dialogar sobre dúvidas, preocupações e problemas de saúde bucal; receptividade ao adentrar o serviço odontológico na APS; e recebimento de informações sobre condição de saúde bucal na APS. As demais variáveis não permaneceram associadas de forma significativa após o teste de seleção de atributos. Conclusão: A qualidade do acolhimento dos serviços de saúde bucal está significativamente relacionada à humanização do atendimento, receptividade do cirurgião-dentista e equipe e aos processos de escuta e orientação.
\end{abstract}

Palavras-chave: acolhimento; avaliação de serviços de saúde; saúde bucal.

\begin{abstract}
Introduction: This study aimed to analyze the quality of care in oral health services regarding its responsiveness to users' needs. Methodology: This cross-sectional and quantitative study included users of the public dental service in the context of Primary Health Care (PHC) in municipalities of three different Brazilian states $(n=1312)$. Data collection occurred through interviews within health facilities. The study included 15 variables related to the reception process, which were preliminarily analyzed with chi-square tests. The variables associated with the outcome 'responsiveness to users' needs' were analyzed through logistic regression and feature selection test. Results: Negative assessments of full attention to users' needs were significantly associated with: care provided by the dental surgeon and staff; willingness to discuss doubts, concerns and oral health problems; initial receptivity in the $\mathrm{PHC}$ dental service; and provision of information on oral health in the PHC. The other variables were not significantly associated with the outcome variable after the feature selection test. Conclusion: the quality of reception in oral health services is significantly related to the humanization of care, responsiveness of dentists and staff and the processes of listening and providing guidance.
\end{abstract} Keywords: reception of users; health service evaluation; oral health.

\footnotetext{
1 Departamento de Odontologia Infantil e Social, Faculdade de Odontologia de Araçatuba, Universidade Estadual Paulista (UNESP) - Araçatuba, SP, Brasil. ${ }^{2}$ Departamento de Odontologia, Universidade Estadual de Ponta Grossa (UEPG) - Ponta Grossa (PR), Brasil. Trabalho realizado nas unidades básicas de saúde dos municípios de Ponta Grossa (PR), Araçatuba (SP) e Aquidauana (MS), Brasil. Endereço para correspondência: Suzely Adas Saliba Moimaz - Rua José Bonifácio, 1193, Seção de Pós-graduação - Vila Mendonça - CEP: $16015-050$ Araçatuba (SP), Brasil - Email: sasaliba@foa.unesp.br Fonte de financiamento: Ministério da Saúde e CNPq (Processo nº 403176/2012-3). Conflito de interesses: nada a declarar.
} 


\section{INTRODUÇÃO}

No Brasil, o termo acolhimento encontra ressonância enquanto estratégia em saúde a partir da implementação da Política Nacional de Humanização - $\mathrm{PNH}^{1}$, que traz como dimensão central o repensar do modelo de gestão no Sistema Único de Saúde (SUS). Sob essa óptica, o acolhimento compreende desde a entrada do usuário no sistema de saúde e a responsabilização integral de suas necessidades até a atenção resolutiva de todos os seus problemas. Frente a essa determinação ampla, o acolhimento pode então provocar a reorganização do serviço ao atender positivamente os entraves em saúde dos usuários.

Na perspectiva do cuidado integral em saúde, acolhimento articula-se intimamente com acesso e implementação de práticas nos serviços de saúde que buscam a resolutividade ${ }^{2,3}$. Como território fértil de encontro entre trabalhador e usuário, o acolhimento torna-se então uma estratégia que potencializa o acesso e permite priorizar grupos de risco, social e epidemiológico. Em cada localidade essa prática de cuidado apresenta singularidades, considerando os modos de viver, de agir, as condições socioeconômicas de cada usuário ${ }^{4}$, e ainda o grau de envolvimento, organização e recursos disponíveis às equipes de saúde 5 .

Diante dessa realidade entende-se que o acolhimento em saúde bucal se apresenta como um dispositivo capaz de nortear a organização e o planejamento das atividades desenvolvidas no SUS, de forma mais eficiente e produtiva, transformando-se em um importante instrumento de gestão. De forma contra-hegemônica, o acolhimento deve acontecer de maneira contínua e dinâmica em todos os locais e momentos do serviço, e não somente como uma forma refeita de recebimento da demanda espontânea, de urgências e emergências.

Para Silva e Romano, o acolhimento vincula-se com a gestão do cuidado e o processo de trabalho, entendendo-o como múltiplo e singular em sua dimensão de produção da saúde. No entanto, poucos são os estudos que se dedicam à abordagem do acolhimento de forma contextual e sistêmica, limitando-se, em sua maioria, a uma análise segmentada dessa estratégia, com abordagem puramente qualitativa.

O objetivo no presente estudo foi analisar a qualidade do acolhimento no serviço de saúde bucal, considerando sua capacidade de resposta às necessidades do usuário.

\section{METODOLOGIA}

O presente estudo transversal, exploratório e descritivo, com abordagem metodológica quantitativa, recebeu aprovação do Comitê de Ética em Pesquisas com Seres Humanos de uma instituição de ensino superior (Parecer n. 353.893/2013), e contou com financiamento do Ministério da Saúde e apoio do Conselho Nacional de Desenvolvimento Científico e Tecnológico - CNPq através da Chamada MCTI/CNPq/MS - SCTIE - Decit n. 10/2012 - Pesquisa em Saúde Bucal (Processo n. 403.176/2012-3).

O universo amostral foi composto por usuários da rede pública odontológica no âmbito da atenção primária à saúde que haviam recebido atendimento há, no máximo, um ano da realização da entrevista e maiores de 18 anos.

Três municípios com distintos aportes populacionais e localizados em diferentes estados brasileiros fizeram parte do estudo. Foi contemplada como local de investigação a totalidade de suas unidades de saúde urbanas, que prestavam serviço odontológico e estavam regularmente em funcionamento no período de coleta. A distribuição de usuários investigados por unidade de saúde foi estratificada, em dias e períodos alternados de funcionamento das unidades de saúde, com o objetivo de ampliar sua representatividade e acessar a variedade de usuários.

No cálculo amostral, considerou-se a população adulta estimada em cada município estudado, $50 \%$ de prevalência para cada possibilidade de resposta (sim/não) à pergunta que continha a variável desfecho, precisão de 5\%, intervalo de confiança de $95 \%$ e efeito de desenho 1 . O cálculo foi determinado através do software Epi.Info 7.1.4. O total calculado para cada município foi de 384 indivíduos. Aos municípios de maior porte, foram acrescidos $20 \%$ ao valor calculado, resultando na amostra final de 1.312 adultos.

A obtenção dos dados foi realizada empregando-se formulário próprio e a coleta foi conduzida por pesquisadores treinados, através de entrevistas individuais, com duração média de 15 minutos.

Os dados decorrentes das questões e suas associações com a variável desfecho foram analisados preliminarmente realizando-se análises bivariadas com a aplicação dos testes qui-quadrado. Posteriormente, as variáveis associadas ao atendimento das necessidades em saúde bucal em nível de significância menor que $5 \%(\mathrm{p}<0,05)$ foram avaliadas através da regressão logística para que a partir das razões de chances fossem verificadas as magnitudes das associações. A regressão foi ajustada da avaliação negativa do atendimento das necessidades em saúde bucal.

Ainda, a fim de verificar as relações de maior força das variáveis independentes à variável desfecho, realizou-se o teste de seleção de atributos utilizando o algoritmo Cfs com validação cruzada de 10 Fold $^{6}$ no ambiente WEKA.

\section{RESULTADOS}

Quando investigados sobre o atendimento de suas necessidades em saúde bucal, $76,4 \%$ dos usuários expuseram que todas as suas necessidades foram atendidas, $21,3 \%$ responderam negativamente, e somente $2,3 \%$ recusaram-se a emitir opinião sobre o assunto, sendo descartados para efeito de análise de resultados. Outras questões também apresentaram algumas não 
respostas, levando a pequenas diferenças no número absoluto de respondentes, não significativas.

A Tabela 1 expressa a análise entre a variável desfecho acolhimento e as demais variáveis que contemplam o processo. As variáveis descritas acerca do nível primário de atenção que apresentaram associação com o atendimento das necessidades em saúde bucal foram: receptividade ao adentrar o serviço; horário de atendimento do cirurgião-dentista; tempo de espera para agendar a consulta; tempo aguardado para atendimento; recebimento de informações sobre condição de saúde bucal;

Tabela 1. Análises bivariadas entre atendimento das necessidades em saúde bucal e variáveis de acolhimento do serviço odontológico na Atenção Primária (APS) e Secundária em Saúde (ASS)

\section{Variável}

\section{Receptividade ao adentrar o serviço odontológico na APS}

Boa

Ruim

Horário de atendimento do cirurgião- dentista na APS

Adequado

Inadequado

Tempo de espera para agendar a consulta na APS

Não demorado

Demorado

Forma de agendamento da consulta na APS

Com hora marcada

Sem hora marcada

Tempo aguardado para o atendimento na APS

Não demorado

Demorado

Recebimento de informações sobre condição de saúde bucal na

APS

Sim

Não

Disponibilidade para dialogar sobre dúvidas, preocupações e

problemas de saúde bucal

Sim

Não

Interesse, demonstrado pelo cirurgião-dentista e equipe da APS, pela saúde bucal do usuário

Sim

Não

Atendimento do cirurgião-dentista e equipe

Bom

Ruim

Necessidade de encaminhamento para a atenção especializada

Sim

Não

Explicação dos profissionais da APS sobre o tratamento a ser realizado na atenção especializada

Sim

Não

Conquista da consulta especializada

Sim

Não

Tempo para agendar a consulta especializada

Não demorado

Demorado

Facilidade em falar com o profissional da APS após término do tratamento

Não
Atendimento das necessidades em saúde bucal

Posi

$\begin{array}{lccc}\text { Positiva } & \text { Negativa } & \text { Total } & \text { p valor } \\ n(\%) & n(\%) & n(\%) & n(\%)\end{array}$

$\begin{array}{rrrr}949(85) & 161(15) & 1110(100) & <\mathbf{0 , 0 0 0 1} \\ 53(31) & 118(69) & 171(100) & \end{array}$

$954(83)$

$45(38)$

202(17)

1156(100)

$<0,0001$

117(100)

506(68)

72(62)

749(100)

538(100)

443(77)

243(32)

45(8)

579(100)

0,2061

559980)

136(23)

702(100)

480(70)

143(20)

688(100)

586(100)

$<0,0001$

517(88)

69(12)

915(83)

184(17)

1099(100)

88(53)

165(100)

$<0,0001$

77(47)

873(84)

171(16)

1044(100)

226(100)

$<0,0001$

120(53)

106(47)

978(84)

192(16)

1170(100)

0,9767

24(22)

87(78)

111(100)

968(85)

245(78)

758(79)

169(83)

181(81)
34(25)

175(15)

104(75)

1143(100)

138(100)

71(22)

316(100)

0,7391

206(21)

964(100)

45(90)

35(17)

5(10)

204(100)

50(100)

42(19)

223(100)

0,1016

30(70)

13(30)

43(100)

$<0,0001$
128(84)

24(16)

152(100)

100(100)

72(72)

$28(28)$

0,0289 $\mathbf{0 , 0 0 3 7}$ 
disponibilidade para dialogar sobre suas dúvidas, preocupações e problemas de saúde bucal; atendimento do cirurgião-dentista e equipe; e facilidade em falar com o profissional após término do tratamento.

Dos usuários que precisaram ser encaminhados para a atenção secundária, $(\mathrm{n}=316), 78 \%$ tiveram suas necessidades em saúde bucal atendidas, sendo o tempo para agendar a consulta nesse segmento de atenção a variável que apresentou implicações no atendimento das necessidades em saúde bucal.

A Tabela 2 mostra os resultados da regressão logística. A avaliação negativa do atendimento das necessidades esteve estatisticamente associada ao: atendimento do cirurgião-dentista e equipe ( $\mathrm{OR}=6,30)$; disponibilidade para dialogar sobre dúvidas, preocupações e problemas de saúde bucal $(\mathrm{OR}=2,83)$; receptividade ao adentrar o serviço odontológico na APS $(\mathrm{OR}=1,98)$; horário de atendimento do cirurgião-dentista na APS (OR $=1.6582$ ); facilidade em falar com o profissional da APS após término do tratamento $(\mathrm{OR}=1,40)$; e recebimento de informações sobre condição de saúde bucal na APS $(\mathrm{OR}=1,25)$. As demais variáveis não permaneceram associadas de forma significativa após a regressão logística.

Ainda, ao realizar o teste de seleção de atributos, verificou-se que as variáveis mais fortemente relacionadas ao atendimento das necessidades em saúde bucal foram: o atendimento do cirurgião-dentista e equipe (100\%); a disponibilidade para dialogar sobre dúvidas, preocupações e problemas de saúde bucal (100\%); a receptividade ao adentrar o serviço odontológico na APS (70\%); e o recebimento de informações sobre condição de saúde bucal (50\%), respectivamente.

\section{DISCUSSÃO}

É considerável o tempo que o governo brasileiro vem dedicando à implantação e ao incentivo de estratégias de fortalecimento do acolhimento nos serviços de saúde, em especial na atenção primária ${ }^{1,7,8}$. Contudo, muitos estudos mostram que a equipe profissional e os gestores locais dos serviços ainda têm grandes dificuldades em compreender o que realmente significa o acolhimento e quais são as etapas e estratégias que norteiam esse processo ${ }^{9-12}$. Neste sentido, a concepção histórica e epistemológica das equipes de saúde bucal e seu perfil predominantemente tecnicista e desarticulado ampliam os desafios da implementação do acolhimento.
No presente estudo buscou-se verificar os aspectos, na óptica do usuário, que qualificam o processo de acolhimento, a fim de auxiliar os profissionais a tornarem o atendimento verdadeiramente mais acessível, resolutivo e humanizado.

Ao avaliar diferentes marcadores do processo de acolhimento no serviço público odontológico brasileiro, verificou-se que $o$ atendimento prestado pela equipe de saúde bucal e a disponibilidade de seus membros para dialogar sobre dúvidas, preocupações e problemas de saúde bucal foram os fatores que mais impactaram na sua qualificação.

Deste modo, considera-se que a capacidade de resposta às necessidades do usuário se traduz de maneira efetiva e se amplia à medida que a prática profissional perpassa por uma busca prudente de reconhecer, para além das demandas explícitas, as necessidades integrais, interiores e subjetivas do indivíduo no concernente à sua saúde $e^{9,13-18}$.

Para tanto, as ações desenvolvidas durante o atendimento devem se deslocar do contexto biomédico reducionista de realização de procedimento clínico, para um processo de trabalho centrado no vínculo profissional/paciente; na promoção da saúde; no fortalecimento da autonomia; na cooparticipação e responsabilização ativa dos problemas em saúde $e^{11-13,15}$.

A utilização de tecnologias leves como escuta ativa, diálogo coparticipativo, empatia, respeito, solidariedade, estabelecimento de confiança, elo de afetividade, credibilidade, valorização e visão integral do outro, configuram-se como fundamentais para garantia de um atendimento humanizado e de qualidade ${ }^{9,12-15,17,19}$, por vezes sobrepondo-se à escassez de recursos materiais e humanos ${ }^{19}$.

Ainda, ao atrelar as tecnologias leves a aspectos singulares do indivíduo, da família e da comunidade na condução do atendimento, eleva-se a probabilidade de garantir resultados mais eficientes no processo atenção em saúde ${ }^{13,16,19,20}$.

Contudo, de acordo com estudos ${ }^{9,16-18,20}$, esta prática só será efetiva se os sentimentos e afetos forem mobilizados, por ambos, usuário e profissional, e o serviço de saúde estabelecer essa prática como rotina prioritária. Sendo assim, para garantir um serviço de qualidade que atenda as necessidades em saúde bucal dos usuários é preciso qualificar as políticas de educação na saúde, de educação permanente e humanização, a partir da realidade vivida pelos atores envolvidos, tendo os enfrentamentos

Tabela 2. Razões de chances ajustadas da avaliação negativa do atendimento odontológico, segundo variáveis independentes

\section{Variável}

Receptividade ao adentrar o serviço odontológico na APS

Horário de atendimento do cirurgião-dentista na APS

Recebimento de informações sobre condição de saúde bucal na APS

Disponibilidade para dialogar sobre dúvidas, preocupações e problemas de saúde bucal

Atendimento do cirurgião-dentista e equipe

Facilidade em falar com o profissional da APS após término do tratamento
Odds Ratio (OR)

1,98

1,66

1,25

2,83

6,30

1,40 
cotidianos do trabalho e as experiências desses atores como base de problematização e de mudança.

A forma com que o usuário foi recebido ao adentrar o serviço odontológico na APS no presente estudo também se destacou como um fator que impacta significativamente na qualificação do acolhimento. Os usuários que relataram descontentamento com a receptividade dos profissionais da saúde apresentaram 1,98 vezes menos chances de o serviço atender as suas necessidades em saúde bucal.

Estudos apontam serem frequentes a impessoalidade e a falta de vínculo dos profissionais da saúde durante o processo de recepção ${ }^{3,13,14}$. O usuário que chega à unidade de saúde quer ser tratado de acordo com a sua individualidade e ter assegurado o direito de falar sobre os seus problemas e encaminhamentos, segundo a sua capacidade de compreensão. Muitas vezes, o simples ouvir e chamar o usuário pelo nome, demonstrando interesse pelo que foi dito, já trazem grande alívio para suas demandas interiores ${ }^{14} \mathrm{e}$ fazem com que ele se sinta realmente respeitado no local onde busca por atenção.

Torna-se então evidente que atitudes de respeito, atenção, cordialidade, gentileza e escuta ativa devam ser valorizadas e intensificadas por todos os interlocutores que têm contato com usuário, desde a sua inserção no sistema de saúde. Essas atitudes fazem com que o usuário se sinta valorizado e estimulado a buscar soluções para os seus problemas e possa desenvolver uma autonomia cognitiva, que garanta o seu empoderamento na utilização do serviço de saúde bucal.

Trazendo novamente à tona essa espécie de desagregação coletiva de condutas de solidariedade, sabe-se que muitos profissionais experenciam, em sua prática diária de trabalho, tensões provenientes de fontes como a dor e o sofrimento, o receio de falhar, a relação com sujeitos de difícil relacionamento, entre outras. No entanto, não há outra forma de concretizar uma rede de construção permanente de cidadania sem a discussão sobre o modo de olhar cada sujeito e cada história de vida.

Assumir a saúde como capital social, além de biológico, é também (re)configurar o padrão de vínculo com usuários, possibilitando-lhes participar como cogestores de suas ações e intervenções em saúde.

Também neste sentido, o outro aspecto determinante na qualificação do acolhimento para o usuário foi a possibilidade de receber informações sobre a sua condição de saúde bucal. Quando o usuário é desqualificado no saber que possui sobre si mesmo, ele terá pouca ou nenhuma participação na construção de seu projeto terapêutico ${ }^{11} \mathrm{e}$, por conseguinte, reduzidas chances de ter suas prioridades assistidas.

Deste modo, é fundamental que profissionais da saúde proporcionem ao usuário, de forma integrada e multiprofissional, um espaço aberto para o diálogo sobre a sua condição de saúde, desde a construção de seu projeto terapêutico, para que ele assuma o papel de sujeito ativo na tomada de decisões. Só assim será possível levar o usuário ao encontro de suas potencialidades, à construção de atos terapêuticos corresponsabilizados e coautorais, ao empoderamento para o enfrentamento de problemas e à oferta de um cuidado mais adequado e condizente com sua realidade e expectativa.

Em face ao exposto, torna-se nítido que as questões relacionadas aos processos organizacionais intrínsecos dos serviços de saúde bucal como a forma e o tempo para conseguir uma consulta em nível primário ou especializado, ou ainda o horário que o cirurgião-dentista trabalha, ocupem destaque secundário no processo de qualificação do acolhimento, em detrimento das relações estritas de vínculo, comunicação, receptividade entre usuários e profissional da saúde.

Neste sentido, é fundamental que haja a sensibilização de gestores para o desenvolvimento de estratégias vinculadas à Política Nacional de Educação Permanente que fomentem a capacitação de profissionais da saúde, a incorporação de novos conhecimentos e tecnologias leves e o desenvolvimento contínuo de habilidades comunicacionais.

Deste modo, espera-se que esta pesquisa possa auxiliar gestores locais de saúde no repensar de suas estratégias de atenção à saúde bucal, com ênfase no processo de acolhimento, que em sua maioria é equivocadamente apenas direcionado à organização da demanda espontânea ${ }^{10}$. A lógica da implementação do acolhimento deve ser baseada na utilização de tecnologias leves que agreguem o vínculo e a boa relação profissional paciente durante todo o processo de trabalho. Os profissionais devem ser capacitados e avaliados constantemente para atuação efetiva de uma prática capaz de acolher, escutar e dar respostas às suas necessidades, resolver os problemas ou orientar e apoiar em busca de soluções para suas demandas, dentro da própria unidade de saúde ou em outros serviços. Ainda, para maior eficácia, devem-se fortalecer os processos de contratação em caráter efetivo de profissionais da saúde a fim de propiciar o profícuo estabelecimento de vínculo ${ }^{19}$.

\section{CONSIDERAÇÕES FINAIS}

O estudo propiciou um aprofundamento sobre a qualificação do acolhimento no serviço público odontológico brasileiro, por meio da sua capacidade de resposta às necessidades do usuário. Os elementos centrais da qualificação foram o atendimento odontológico satisfatório, a disponibilidade em dialogar sobre dúvidas, preocupações e problemas de saúde bucal, a receptividade ao adentrar o serviço odontológico na APS, a orientação e o empoderamento sobre condição de saúde bucal. 


\section{REFERÊNCIAS}

1. Brasil. Ministério da Saúde. Secretaria de Atenção à Saúde. Humaniza SUS: Documento base para gestores e trabalhadores do SUS. 4 ed. Brasília, DF: Ministério da Saúde; 2008.

2. Franco TB, Bueno WS, Merhy EE. O acolhimento e os processos de trabalho em saúde: o caso de Betim, Minas Gerais, Brasil. Cad Saude Publica. 1999;15(2):345-53. PMid:10409787. http://dx.doi.org/10.1590/ S0102-311X1999000200019.

3. Souza ECF, Vilar RLA, Rocha NSPD, Uchoa AC, Rocha PM. Acesso e acolhimento na atenção básica: uma análise da percepção dos usuários e profissionais de saúde. Cad Saúde Publica. 2008;24(Supl 1):100-10.

4. Silva TF, Romano VF. Sobre o acolhimento: discurso e prática em Unidades Básicas de Saúde do município do Rio de Janeiro. Saúde Debate. 2015;39(105):363-74. http://dx.doi.org/10.1590/0103-110420151050002005.

5. Almeida PF, Fausto MC, Giovanella L. Fortalecimento da atenção primária à saúde: estratégia para potencializar a coordenação dos cuidados. Rev Panam Salud Publica. 2011;29(2):84-95. PMid:21437365. http://dx.doi. org/10.1590/S1020-49892011000200003.

6. Hall MA. Correlation-based feature subset selection for machine learning [thesis]. Hamilton, New Zealand: University of Waikato; 1999.

7. Brasil. Ministério da Saúde. Secretaria de Atenção à Saúde. Departamento de Atenção Básica. Política nacional de atenção básica. Brasília: Ministério da Saúde; 2006

8. Brasil. Ministério da Saúde. Portaria n ${ }^{\circ} 2.488$, de 21 de outubro de 2011. Aprova a Política Nacional de Atenção Básica, estabelecendo a revisão de diretrizes e normas para a organização da Atenção Básica, para a Estratégia Saúde da Família (ESF) e o Programa de Agentes Comunitários de Saúde (PACS). Diário Oficial da União. Brasília, 2011.

9. Nery SR, Nunes EF, Carvalho BG, Melchior R, Baduy RS, Lima JVC. Acolhimento no cotidiano dos auxiliares de enfermagem nas Unidades de Saúde da Família, Londrina (PR). Cien Saude Colet. 2009;14(Supl 1):1411-9. PMid:19750350. http://dx.doi.org/10.1590/S1413-81232009000800014.

10. Baraldi DC, Souto BGA. A demanda do Acolhimento em uma Unidade de Saúde da Família em São Carlos, São Paulo. Arq Bras Ciênc Saúde. 2011;36(1):10-7.

11. Mitre SM, Andrade EIG, Cotta RMM. O acolhimento e as transformações na práxis da reabilitação: um estudo dos Centros de Referência em
Reabilitação da Rede do Sistema Único de Saúde em Belo Horizonte, MG, Brasil. Cien Saude Colet. 2013;18(7):1893-902. PMid:23827893. http:// dx.doi.org/10.1590/S1413-81232013000700004.

12. Neves M, Pretto SM, Ely HC. Percepções de usuários e trabalhadores de saúde sobre a implantação do acolhimento em uma unidade de saúde em Porto Alegre-RS, Brasil. Rev Odontol UNESP. 2013;42(5):364-71. http:// dx.doi.org/10.1590/S1807-25772013000500008.

13. Schimith MD, Lima MADS. Acolhimento e vínculo em uma equipe do Programa Saúde da Família. Cad Saude Publica. 2004;20(6):1487-94. PMid:15608849. http://dx.doi.org/10.1590/S0102-311X2004000600005.

14. Coelho MO, Jorge MSB. Tecnologia das relações como dispositivo do atendimento humanizado na atenção básica à saúde na perspectiva do acesso, do acolhimento e do vínculo. Cien Saude Colet. 2009;14(Supl. 1):1523-31. PMid:19750362. http://dx.doi.org/10.1590/S1413-81232009000800026.

15. Lima TJV, Arcieri RM, Garbin CAS, Moimaz SAS, Saliba O. Humanização na atenção básica de saúde na percepção de idosos. Saúde Soc. 2014;23:265276.

16. Lima TJV, Arcieri RM, Garbin CAS, Moimaz SAS. Humanização na atenção à saúde do idoso. Saúde Soc. 2010;19:866-877.

17. Jorge MSB, Pinto DM, Quinderé PH, Pinto AG, Sousa FS, Cavalcante CM. Promoção da Saúde Mental - Tecnologias do Cuidado: vínculo, acolhimento, co-responsabilização e autonomia. Cien Saude Colet. 2011;16(7):3051-60. PMid:21808893. http://dx.doi.org/10.1590/S1413-81232011000800005.

18. Sena ALC, Ferreira LN, Oliveira RS, Kozmhinsky VMR. Acolhimento e satisfação do usuário na estratégia de saúde da família: uma experiência de êxito. Rev APS. 2015;18(2):134-40.

19. Moimaz SAS, Lima AMC, Garbin CAS, Corrente JE, Saliba NA. Avaliação do usuário sobre o atendimento odontológico no Sistema Único de Saúde: uma abordagem à luz da humanização. Cien Saude Colet. 2016;21(12):387987. http://dx.doi.org/10.1590/1413-812320152112.12672015.

20. Santos AM, Assis MMA. Da fragmentação à integralidade: construindo e (des)construindo a prática de saúde bucal no Programa de Saúde da Família (PSF) de Alagoinhas, BA. Cien Saude Colet. 2006;11(1):53-61. http://dx.doi.org/10.1590/S1413-81232006000100012.

Recebido em: Ago. 14, 2016 Aprovado em: Jan. 26, 2017 